\title{
Testing the addictive appetite model of binge eating: The importance of craving, coping, and reward enhancement
}

Short Title: Testing the addictive appetite model of binge eating

Monica Leslie* $*_{1}$

Robert Turton*1

Emilee Burgess2

Bruno Nazar $\uparrow 1,3$

Janet Treasure $\uparrow 1$

* Joint first author

$\dagger$ Joint senior author

1 Section of Eating Disorders, Department of Psychological Medicine, Institute of Psychiatry, Psychology and Neuroscience, King's College London, London, UK

2 Department of Psychology, University of Alabama at Birmingham, USA

3 Federal University of Rio de Janeiro - Institute of Psychiatry (IPUB - UFRJ), Rio de Janeiro, Brazil

Corresponding Author:

Monica Leslie

Section of Eating Disorders,

Department of Psychological Medicine

103 Denmark Hill, London, SE5 8AF, King's College London

Email address: monica.leslie@kcl.ac.uk

Acknowledgements: The authors declare no conflict of interest. ML was supported by grants from the Swiss Fund for Anorexia Nervosa (Grant 43-14) and a King's Health Partners Challenge Fund (Grant R1405174). JT was supported by the National Institute for Health Research (NIHR) Mental Health, the BRC Obelix framework, and King's College London. RT was supported by the Medical Research Council and Psychiatry Research Trust (PCPTAAR). 


\begin{abstract}
In the current study, we examine components of the "addictive appetite" model of recurrent binge eating. Specifically, we tested the influence of addictive processes and the influence of emotional regulation processes on recurrent binge eating behaviour. We recruited 79 women in total for the current study; 22 with bulimia nervosa, 26 weight-matched lean comparison women, 15 women with binge eating disorder, and 16 weight-matched overweight/obese comparison women. Participants completed questionnaire assessments of food craving and motivations for eating. Compared to weight-matched comparison women, women with binge-type eating disorders endorse significantly greater levels of food craving, eating for purposes of coping, and eating for purposes of reward enhancement. A cluster analysis revealed that these three traits distinguish women with binge-type eating disorders from weight-matched comparison women. These findings provide support for the addictive appetite model of binge eating behaviour, and highlight addictive and emotional regulation processes as potential targets for treatment.
\end{abstract}

Key Words: Bulimia Nervosa; Binge Eating Disorder; Food Addiction; Research Domain Criteria 
The trans diagnostic model of eating disorders has played a dominant role in informing psychological treatment for adults with bulimia nervosa and binge eating disorder over the last several decades (Fairburn, Cooper, \& Shafran, 2003). However, treatment informed by the trans diagnostic approach is not effective for many individuals with bulimia nervosa, with only 30-50\% achieving abstinence from binge-purge behaviours by the end of treatment (Hay, 2013; National Institute for Health and Clinical Excellence, 2017), and 68\% achieving remission at 9-year follow-up (Eddy et al., 2016).

An updated model of recurrent binge eating behaviour has therefore recently been proposed (Treasure, Leslie, Chami, \& Fernández-Aranda, 2018), which addresses the dysregulation of appetite in bulimia nervosa and binge eating disorder not previously highlighted within the trans diagnostic model. One of the key factors proposed by this updated "addictive appetite" model of recurrent binge eating behaviour is the increased incentive salience of food cues and habitual response patterns to palatable food cues, which heighten desire and craving for these foods (Berridge, 2007). At a neural level, this pattern of heightened response to food cues and subdued responding to the receipt of pleasant tastes bears resemblance to substance use disorders, in which a similar pattern of neural responding has been observed in relation to drug cues (Gearhardt et al., 2011). For the purposes of the current paper, we define food craving as being characterised by intense desire or urge to eat a particular type of food, where that desire correspond to a specific flavour and texture (Pelchat, 2002). "Craving", in its current usage, can therefore be contrasted to both generalised hunger that does not necessarily drive behaviour towards a specific type of food, and to mild preferences for one type of food over another.

There is also thought to be a down-regulation of the response to other rewards in bulimia nervosa and binge eating disorder, such that basal levels of low affect are common in this population (Volkow, Wise, \& Baler, 2017). This low affect thus contributes to heightened likelihood of compensatory reward-seeking, specifically in the form of palatable tastes 
(Treasure et al., 2018). This model of binge eating behaviour has previously received support from ecological momentary assessment studies, which found that participants reported greater levels of low affect immediately prior to binge eating episodes (Haedt-Matt \& Keel, 2011). However, relatively little previous research has explored the relationship between individuals' motivations for eating and eating disorder pathology (Burgess, Turan, Lokken, Morse, \& Boggiano, 2014).

The aim of this study was to test two maintenance processes proposed within the addictive appetite model of binge eating: eating due to enhanced incentive salience and eating for emotion regulation. Our hypotheses were as follows:

1. People with BN and BED would show higher levels of craving for food than weightmatched controls.

2. People with BN and BED would endorse eating palatable food as a method of coping with distress and as a means of enhancing mood.

3. Food craving, eating for emotional coping, and eating for reward enhancement would distinguish individuals with binge-type eating disorders from weight-matched controls.

\section{Methods}

\section{Participants}

Seventy-nine women were recruited through flyers and e-mail circulars at King's College London, as well as through eating disorder clinics in London. Twenty-six women met criteria for the lean control group, 22 women met DSM-5 criteria for bulimia nervosa, 15 women met DSM-5 criteria for binge eating disorder, and 16 women were included in the overweight/obese control group. Of the women with bulimia nervosa or binge eating disorder, 25 had received treatment for their eating disorder within six months prior to the study. Further details regarding the type of treatment participants received are presented in Supplementary

Table 1. Of the participants with BN or BED, 15 participants had a comorbid diagnosis of a 
depressive disorder, 8 participants had comorbid generalised anxiety, 1 participant had a comorbid diagnosis of social anxiety disorder, two participants had borderline personality disorder, two participants had post-traumatic stress disorder, and 1 participant had obsessivecompulsive disorder.

The non-eating disorder comparison groups were age- and weight-matched with the groups with eating disorders as far as possible. Ethical approval for the study was granted by the Hampstead Research Ethics Committee (reference number: 14/LO/2166). All participants voluntarily consented to take part in the study.

Inclusion criteria for the study required participations' age to be between 18 and 65 years old. Participants in the eating disorder sample were required to meet DSM-5 diagnostic criteria for BN or BED (American Psychiatric Association, 2013). Healthy control participants were required to have a body mass index (BMI) of at least 18.5 , and have no history of an eating disorder. Exclusion criteria included: current substance abuse, history of an abnormal neurological condition, acute suicidality, and severe co-morbidity (e.g., active psychosis). Participants were screened using the Structured Clinical Interview for DSM-5 (SCID) (American Psychiatric Association, 2013). Screening was conducted either by a psychiatrist or graduate-level psychology student who had received specific training in correct use of the SCID. Demographic descriptive statistics for each sample of participants are reported in Table 1.

\section{Experimental Design}

After signing informed consent and undergoing screening for eligibility, participants were invited to complete an online survey containing the following battery of measures: the Eating Disorder Examination - Questionnaire version (EDE-Q) (Fairburn \& Beglin, 1994), the Depression and Anxiety Stress Scales (DASS) (Lovibond \& Lovibond, 1995), Food Craving Questionnaire - Trait subscale (FCQ) (Cepeda-Benito, Gleaves, Williams, \& Erath, 2000), and 
the Palatable Eating Motives Scale (PEMS) (Burgess et al., 2014). These online questionnaires were conducted prior to a subsequent battery of tests conducted in the lab, the results of which are reported in a previously published paper (Turton et al., 2018). The PEMS has been described in detail below and all other measures are included within the Supplementary Material. Participants were also asked to confirm their current binge eating frequency and answer demographic questions, including their age and education level (measured as the total number of years spent in education).

Palatable Eating Motives Scale (PEMS). The Palatable Eating Motives Scale (PEMS) (Burgess et al., 2014) yields a measure of the extent to which individuals tend to eat food for reasons other than maintaining metabolic homeostasis (i.e., in response to hunger). The PEMS consists of 19 items answered in the form of a 5-point Likert scale from 1 (Almost Never/Never) to 5 (Almost Always/Always). The PEMS contains four subscales corresponding to different reasons people consume palatable food (Social, Coping, Conformity, and Reward Enhancement). The social subscale measures motivation to eat for social reasons (e.g., when going out for a meal or to a party with friends), the coping subscale measures motivation to eat in order to regulate negative affect (e.g., to distract oneself and/or improve mood after a bad day), the conformity subscale measures motivation to eat in order to due to social pressures to do so (e.g., to fit in with others, or to be liked), while the reward enhancement subscale measures motivation to eat in order to enhance positive experiences or the intrinsic reward of palatable foods. Within the current sample, the total PEMS scale was associated with excellent internal consistency $(\alpha=0.92)$. All subscales of the PEMS were associated with good internal consistency: Social $(\alpha=0.88)$, Coping ( $\alpha=0.95)$, Enhancement $(\alpha=0.85)$, and Conformity $(\alpha=0.89)$.

\section{Statistical Analysis}


In order to account for the potential effect of BMI, the sample with bulimia nervosa was compared against a weight-matched lean comparison group matched in the following analyses, and the sample with binge eating disorder was likewise compared against an overweight/obese comparison group. Each of the above-mentioned variables were compared across groups using a Student's $t$-test. Multiple comparisons were controlled for by setting the False Discovery Rate at 0.05 (Benjamini \& Hochberg, 1995). Exploratory correlation analyses were then conducted to examine relationships between subscales of the EDE-Q, the subscales of the PEMS, BMI, and the FCQ.

A $k$-means cluster analysis was then used to determine whether food craving, eating for emotional coping, and eating for reward enhancement would distinguish individuals with binge-type eating disorders from weight-matched controls. This cluster analysis was conducted using a single-linkage cluster method. Squared Euclidean distance was the chosen distance measure for the cluster analysis. All analyses were performed in SPSS version 23.0.

The data were first analysed for outliers and assumptions of normality. Three outliers $(\mathrm{Z}>|3.0|)$ were observed in the BMI variable, one in the age variable, two in the social subscale of the PEMS, three in the conformity subscale of the PEMS, and one in the binge frequency variable. These values were excluded case-wise from all relevant analyses.

\section{Results}

Demographics. There were no significant differences in age between each sample and its weight-matched comparison group. There were no significant differences in BMI or education between the bulimia nervosa sample and lean control group, although the binge eating disorder group had a significantly higher BMI than the overweight/obese control group $(Z=-2.02, p=.044)$ and spent significantly fewer years in education $(t(28.30)=3.04, p=$ .005). The descriptive statistics and effect size comparisons for these variables are presented in Table 1. 
Eating disorder symptomatology, food craving, depression, anxiety, and eating motivations for each diagnostic group. The descriptive statistics and effect size comparisons for EDE-Q subscales (Eating Concern, Restraint, Weight Concern, Shape Concern, Binge Frequency), FCQ, the PEMS subscales (Social, Conformity, Coping, and Reward Enhancement), and the DASS for each diagnostic group are reported in Table 2. The results of the $t$-tests comparing lean controls with the bulimia nervosa sample on the EDE-Q subscales FCQ, the PEMS subscales, and the DASS are presented in Table 3. Due to excessive skew in the binge eating frequency variable for the lean control sample, this variable was compared to the bulimia nervosa sample using a Mann-Whitney U test. The Mann-Whitney U test for binge frequency revealed that the sample with bulimia nervosa reported a significantly higher binge frequency than the lean control sample $(U=0.50, Z=-6.20, p<.001)$. There were no significant differences regarding the extent to which individuals ate for social reasons measured between these two samples. The sample with bulimia nervosa had significantly higher levels of depression, anxiety, and stress (as measured by the DASS), significantly higher scores on all EDE-Q subscales (restraint, eating concern, weight concern, and shape concern), greater levels of food craving, and reported significantly greater tendencies to eat for purposes of coping, reward enhancement, and conformity than the lean control group.

The results of the $t$-tests comparing the overweight/obese control sample with the binge eating disorder sample on the EDE-Q subscales FCQ, the PEMS subscales, and the DASS are presented in Table 4. There were no significant differences between the two groups in eating for purposes of socialising or conformity. However, the binge eating disorder group had significantly higher levels of depression, anxiety, and stress (as measured by the DASS), significantly higher scores on all EDE-Q subscales (restraint, eating concern, weight concern, and shape concern), a significantly greater frequency of binge eating, greater levels of food 
craving, and reported significantly greater tendencies to eat for purposes of coping and reward enhancement.

The results of the $t$-tests comparing the BN sample with the BED sample on the EDEQ subscales FCQ, the PEMS subscales, and the DASS are reported in Table 5. no significant differences in restraint, shape concern, binge frequency, food craving, eating for social purposes, eating for purposes of coping, eating for purposes of reward enhancement, or depression between participants with binge eating disorder versus participants with bulimia nervosa. Although initial comparisons suggested that participants with binge eating disorder had significantly greater levels of eating concern and weight concern in comparison to participants with bulimia nervosa, these differences did not survive after controlling for multiple comparison. Likewise, while initial the initial $t$-test suggested that people with bulimia nervosa had significantly greater levels of eating for purposes of conformity in comparison to participants with binge eating disorder, this analysis did not hold following correction for multiple comparisons.

Components of food craving for each diagnostic group. The descriptive statistics and effect size comparisons for the FCQ subscales are reported for each diagnostic group in Table 6.

The results of the $t$-tests comparing lean controls with the bulimia nervosa sample on the FCQ subscales are presented in Table 7. There were significant differences between the two samples for all subscales. The BN sample had significantly greater scores than the lean control sample on all FCQ subscales.

The results of the $t$-tests comparing the obese/overweight comparison group with the BED sample on the FCQ subscales are presented in Table 8. (The subscale FCQ Preoccupation was excluded from the $t$-tests as the data was skewed in the overweight/obese 
group.) The BED sample had significantly greater scores than the overweight/obese control sample on all FCQ subscales.

Correlations between eating concerns, craving, eating motivations, and binge eating. Correlations between eating concern, food craving, binge eating frequency, and each subscale of the PEMS for individuals with bulimia nervosa are presented in Supplementary Table 2. Trait levels of food craving were strongly positively correlated with eating for reasons of coping and reward enhancement. The different subscales of the EDE-Q were strongly and positively correlated with each other (with the exception of eating concern and restraint, which were not significantly correlated).

Correlations between eating concern, food craving, binge frequency, and each subscale of the PEMS for individuals with binge eating disorder are presented in Supplementary Table 3. As in bulimia nervosa, most subscales of the EDE-Q were strongly and positively correlated with each other (with the exception of restraint and weight concern). Trait levels of food craving were strongly and positively correlated with eating for emotional coping, reward enhancement, and conformity.

Cluster analysis. We next conducted a $k$-means cluster analysis for all participants including the variables FCQ, PEMS-Coping, and PEMS-Enhancement, where the number of clusters to be identified was set to two. Between-subjects ANOVAs subsequently revealed significant differences between the two clusters on the FCQ $(F=371.24, d f=99, p<.001)$, PEMS-Coping $(F=193.07, d f=99, p<.001)$, and PEMS-Enhancement $(F=48.38, d f=99, p$ $<.001)$.

We then conducted a chi-square test to determine if each of these two clusters contained a significantly different proportion of individuals with and without eating disorders. Cluster 1 contained 42 individuals without an eating disorder, and two with BN or BED. Cluster 2 contained 39 individuals with BN or BED, and 6 without an eating disorder. These proportions 
were significantly different $\left(\chi_{2}=60.39, d f=1, p<.001\right)$, thus indicating that food craving, eating for emotional coping, and eating for reward enhancement significantly distinguished the sample with $\mathrm{BN}$ or BED from the lean and overweight/obese comparison samples.

\section{Discussion}

The current study aimed to test maintenance processes proposed within the addictive appetite model through an assessment of craving and motivations for eating. Our hypotheses were that women with $\mathrm{BN}$ and $\mathrm{BED}$ would endorse significantly greater levels of food craving, and tendencies to eat for purposes of coping and reward enhancement, when compared to weight-matched healthy control participants. Our data supported these hypotheses with strong effect sizes for each comparison. A cluster analysis subsequently supported our hypothesis that food craving, eating for purposes of coping, and eating for purposes of reward enhancement would significantly distinguish women with BN or BED, from the lean and overweight/obese comparison samples of women without an eating disorder.

These findings, drawn from a clinical population of individuals with eating disorders, corroborate results previously reported by (Boggiano et al., 2014), amongst students: such that eating for purposes of coping and reward enhancement was associated with exhibiting recurrent binge eating behaviour, and eating for social purposes was not. Differences in the extent to which individuals reported eating for reasons of conformity was significantly different only between individuals with $\mathrm{BN}$ versus lean controls, and did not differ significantly between the BED group and the overweight/obese control sample. It is not completely clear why this is the case, although one hypothesis may be that in the absence of a visual difference of appearance or weight, the $\mathrm{BN}$ group is more highly motivated to mask their disorder by conforming to the eating behaviours exhibited by others in a social setting. This hypothesis requires further research to corroborate. 
Our findings provide support for the addictive appetite model of bulimia nervosa and binge eating disorder (Treasure, Leslie, Chami, \& Fernández-Aranda) by providing evidence for heightened levels of craving for palatable foods, and the tendency for these individuals to use eating as an emotion regulation strategy. These findings also contribute to the existing field of research testing the affect regulation model of binge eating (Haedt-Matt \& Keel, 2011; Leehr et al., 2015). A meta-analysis of ecological momentary assessment studies by Haedt-Matt and Keel (2011) supported the hypothesis that increased negative mood immediately precipitates binge eating episodes, when compared to both average affect and affect preceding non-binge eating. The findings of the present study similarly highlight that eating for purposes of coping is characteristic of individuals with recurrent loss-of-control binge eating behaviour. Furthermore, a meta-analysis of studies inducing negative affect has also supported a causal link between negative affect and binge eating (Leehr et al., 2015)

The effect of binge eating on mood itself is, however, less clear. Haedt-Matt and Keel (2011), in their meta-analysis found that mood worsened following a binge in a heterogeneous population of binge eaters (including those diagnosed with BN, BED, and self-identified binge eaters). On the contrary, Leehr et al. (2015) reported improved mood following binge eating in people with binge eating disorder. These contradictory findings may be explained by the addictive appetite model of binge eating, which posits that neuroadaptation promotes tolerance for the "liking" hedonic component of tasty foods develops over time (Berridge, 2009; Treasure et al., 2018; Volkow, Fowler, Wang, \& Swanson, 2004). This process co-occurs with progressive increases in "wanting" (incentive salience) of binge foods (Berridge, 2009; Treasure et al., 2018; Volkow et al., 2004). It may therefore be the case that studies using ecological momentary assessment included in the Haedt-Matt and colleagues' meta-analysis fail to capture an immediate reduction in negative urgency occurring during the course of a binge eating episode, while succeeding in the capture of guilt and shame following the binge 
eating episode. This hypothesis receives support from studies demonstrating the influence of negative urgency on binge eating, independent of other facets of negative affect (Anestis, Smith, Fink, \& Joiner, 2009; Racine et al., 2013). Within the addictive appetite model of binge eating, this emotional process is posited to be intrinsically underpinned by the large, repeated incidence of addictive appetite. This repeated addictive appetite over time results in the heightened "wanting" of binge foods and directly contributes to the experience of negative urgency.

The addictive appetite model also highlights the relevance of drawing from theoretical models and effective treatments currently employed within the addictions science literature. Heilig et al. (2010), for example, have proposed that alcohol dependency proceeds from "reward craving" in the early phase of the addiction, to "relief craving" as the addiction proceeds. These constructs are conceptually identical to the motivations for Reward Enhancement and Coping, respectively, proposed in relation to eating within the PEMS. It would therefore be interesting to stratify by duration of illness in future research, to determine whether individuals with bulimia nervosa and binge eating disorder demonstrates a similar progression in eating motivation, from Reward Enhancement to Coping.

The prevalence of emotion-triggered eating within this population further highlights the importance of supporting effective emotional regulation strategies among this population. Indeed, it has previously been found that emotion regulation abilities improve throughout the course of treatment for bulimia nervosa, and especially amongst individuals with a better treatment outcome (Mallorquí-Bagué et al., 2018). It can therefore be hypothesised that improvement in emotional regulation strategies is one of the mediating factors in achieving remission from bulimia nervosa, although further studies measuring the time-course of changes are required to determine whether short-term improvements in emotion regulation strategy precede improves in symptom remission. 
It was interesting to note that, after controlling for multiple comparison, there were no significant differences between women with bulimia nervosa versus women with binge eating disorder on measure of food craving, eating disorder psychopathology, or eating motivations. Within the framework of the addictive appetite model of binge eating, this finding can be explained by the fact that the same neural process is hypothesised to underpin binge eating behaviour in eating population, and therefore operates identically on a functional level in both bulimia nervosa and binge eating disorder (Treasure et al., 2018). These findings therefore highlight commonalities in the psychological profile and maintenance factors of bulimia nervosa and binge eating disorder, suggesting that similar treatment approaches may be useful in treatment both disorders.

Within the current study, the fact that levels of food craving and eating for mood regulation alone can be used to differentiate individuals with BN/BED from weigh-matched controls provides further evidence for the significance of these processes to recurrent binge eating behaviour, even after controlling for overall energy balance, and highlights the importance of targeting emotion regulation in treatment. Individuals with BN and BED may need guidance with managing food-related cues in the environment in the early stages of treatment, given their high levels of craving. The continued development of treatment approaches that weaken automatic stimulus-response associations to palatable food would similarly be useful in supporting recovery (Turton et al., 2018).

Limitations of the current study include the small sample size for each diagnostic group, and the use of self-report measures (which are subject to social desirability bias), and the limitations of the participants' memory and understanding of their motivations for eating. Furthermore, as the current study only included women, the current findings are not generalizable to men with bulimia nervosa or binge eating disorder. The subjective perception 
of the close link between high emotion and eating, however, provides an important indication of the purpose that binge eating serves for women with BN and BED.

We recommend that future studies continue to test other aspects of the addictive appetite model, including the contribution of insulin resistance/sensitivity to the onset of binge eating behaviour. We would also recommend future research to continue to examine the genetic factors accounting for variation in individual risk for binge-eating behaviour. We propose that continued research into the intersection between genetic risk for recurrent binge eating, metabolic dysregulation, and the psychological trait profile of each individual will continue to aid the development of more efficacious and personalised treatment approaches, in line with the burgeoning movement towards 'precision psychiatry' (Fernandes et al., 2017).

In conclusion, the current study identified significantly higher levels of food craving, eating for purposes of emotional coping, and eating for purposes of reward enhancement among women with $\mathrm{BN}$ and $\mathrm{BED}$ versus weight-controlled women without an eating disorder. A cluster analysis subsequently identified that these three traits significantly distinguished women with recurrent binge eating behaviour from the general population. These findings provide further support for the food addiction model of recurrent binge eating behaviour, and highlight the importance of targeting addictive and emotional regulation processes within treatment. 


\section{References}

American Psychiatric Association. (2013). Diagnostic and statistical manual of mental disorders. Arlington: American Psychiatric Publishing.

Anestis, M. D., Smith, A. R., Fink, E. L., \& Joiner, T. E. (2009). Dysregulated eating and distress: Examining the specific role of negative urgency in a clinical sample. Cognitive Therapy and Research, 33(4), 390-397.

Benjamini, Y., \& Hochberg, Y. (1995). Controlling the false discovery rate: a practical and powerful approach to multiple testing. Journal of the royal statistical society. Series $B$ (Methodological), 289-300.

Berridge, K. C. (2007). The debate over dopamine's role in reward: the case for incentive salience. Psychopharmacology (Berl), 191(3), 391-431.

Berridge, K. C. (2009). 'Liking' and 'wanting'food rewards: brain substrates and roles in eating disorders. Physiology \& behavior, 97(5), 537-550.

Boggiano, M. M., Burgess, E., Turan, B., Soleymani, T., Daniel, S., Vinson, L., . . Morse, A. (2014). Motives for eating tasty foods associated with binge-eating. Results from a student and a weight-loss seeking population. Appetite, 83, 160-166.

Burgess, E., Turan, B., Lokken, K. L., Morse, A., \& Boggiano, M. M. (2014). Profiling motives behind hedonic eating. Preliminary validation of the Palatable Eating Motives Scale. Appetite, 72, 66-72.

Cepeda-Benito, A., Gleaves, D. H., Williams, T. L., \& Erath, S. A. (2000). The development and validation of the state and trait food-cravings questionnaires. Behavior Therapy, 31(1), 151-173.

Eddy, K. T., Tabri, N., Thomas, J. J., Murray, H. B., Keshaviah, A., Hastings, E., . . F Franko, D. L. (2016). Recovery From Anorexia Nervosa and Bulimia Nervosa at 22-Year Follow-Up. J Clin Psychiatry. doi:10.4088/JCP.15m10393

Fairburn, C., \& Beglin, S. (1994). Eating Disorders Examination Questionnaire. International Journal of Eating Disorders, 16(4), 363-370.

Fairburn, C., Cooper, Z., \& Shafran, R. (2003). Cognitive behaviour therapy for eating disorders: A "transdiagnostic" theory and treatment. Behaviour research and therapy, 41(5), 509-528.

Fernandes, B. S., Williams, L. M., Steiner, J., Leboyer, M., Carvalho, A. F., \& Berk, M. (2017). The new field of 'precision psychiatry'. BMC medicine, 15(1), 80.

Gearhardt, A. N., Yokum, S., Orr, P. T., Stice, E., Corbin, W. R., \& Brownell, K. D. (2011). Neural correlates of food addiction. Archives of general psychiatry, 68(8), 808-816.

Haedt-Matt, A. A., \& Keel, P. K. (2011). Revisiting the affect regulation model of binge eating: a meta-analysis of studies using ecological momentary assessment. Psychological bulletin, 137(4), 660.

Hay, P. (2013). A systematic review of evidence for psychological treatments in eating disorders: 2005-2012. International Journal of Eating Disorders, 46(5), 462-469.

Heilig, M., Thorsell, A., Sommer, W. H., Hansson, A. C., Ramchandani, V. A., George, D. T., . . . Barr, C. S. (2010). Translating the neuroscience of alcoholism into clinical treatments: from blocking the buzz to curing the blues. Neuroscience \& Biobehavioral Reviews, 35(2), 334-344. 
Leehr, E. J., Krohmer, K., Schag, K., Dresler, T., Zipfel, S., \& Giel, K. E. (2015). Emotion regulation model in binge eating disorder and obesity-a systematic review. Neuroscience \& Biobehavioral Reviews, 49, 125-134.

Lovibond, P. F., \& Lovibond, S. H. (1995). The structure of negative emotional states: Comparison of the Depression Anxiety Stress Scales (DASS) with the Beck Depression and Anxiety Inventories. Behaviour research and therapy, 33(3), 335-343.

Mallorquí-Bagué, N., Vintró-Alcaraz, C., Sánchez, I., Riesco, N., Agüera, Z., Granero, R., . . Fernández-Aranda, F. (2018). Emotion Regulation as a Transdiagnostic Feature Among Eating Disorders: Cross-sectional and Longitudinal Approach. European Eating Disorders Review, 26(1), 53-61.

National Institute for Health and Clinical Excellence. (2017). Eating Disorders: recognition and treatment. NICE Guideline (NG69).

Pelchat, M. L. (2002). Of human bondage: Food craving, obsession, compulsion, and addiction. Physiology \& behavior, 76(3), 347-352. doi:https://doi.org/10.1016/S0031-9384(02)00757-6

Racine, S. E., Keel, P. K., Burt, S. A., Sisk, C. L., Neale, M., Boker, S., \& Klump, K. L. (2013). Exploring the relationship between negative urgency and dysregulated eating: Etiologic associations and the role of negative affect. Journal of abnormal psychology, 122(2), 433.

Treasure, J., Leslie, M., Chami, R., \& Fernández-Aranda, F. Are trans diagnostic models of eating disorders fit for purpose? A consideration of the evidence for food addiction. European Eating Disorders Review, n/a-n/a. doi:10.1002/erv.2578

Treasure, J., Leslie, M., Chami, R., \& Fernández-Aranda, F. (2018). Are trans diagnostic models of eating disorders fit for purpose? A consideration of the evidence for food addiction. European Eating Disorders Review, 26(2), 83-91. doi:10.1002/erv. 2578

Turton, R., Nazar, B. P., Burgess, E. E., Lawrence, N. S., Cardi, V., Treasure, J., \& Hirsch, C. R. (2018). To Go or Not to Go: A Proof of Concept Study Testing Food-Specific Inhibition Training for Women with Eating and Weight Disorders. European Eating Disorders Review, 26(1), 11-21.

Volkow, N. D., Fowler, J. S., Wang, G.-J., \& Swanson, J. M. (2004). Dopamine in drug abuse and addiction: results from imaging studies and treatment implications. $\mathrm{Mol}$ Psychiatry, 9(6), 557.

Volkow, N. D., Wise, R. A., \& Baler, R. (2017). The dopamine motive system: implications for drug and food addiction. Nature Reviews Neuroscience, 18(12), 741. 